\title{
ASSEMBLEIAS DE FORMIGAS (HYMENOPTERA: FORMICIDAE) RESPONDEM AO PROCESSO DE RECUPERAÇÃO DE ÁREAS DE PRESERVAÇÃO PERMANENTE?
}

DO ANT ASSEMBLIES (HYMENOPTERA: FORMICIDAE) PREDICT

THE RECOVERY PROCESS OF PERMANENT PRESERVATION AREAS?

\section{Junir Antônio Lutinski iD}

Doutor em Biodiversidade Animal, Universidade Federal de Santa Maria. Professor, Programa de Pós-Graduação em Ciências da Saúde, Universidade Comunitária da Região de Chapecó (Unochapecó) - Chapecó (SC), Brasil.

\section{Carin Guarda}

Doutoranda, Programa de

Pós-Graduação em Ciências da Saúde, Unochapecó - Chapecó (SC), Brasil.

\section{Cladis Juliana Lutinski iD}

Mestre em Ciências Ambientais, Unochapecó. Bióloga, Laboratório de Biologia, Universidade Federal da Fronteira Sul - Chapecó (SC), Brasil.

\section{Fernanda Emanuela} Dorneles (iD)

Graduanda em Ciências Biológicas, Unochapecó - Chapecó (SC), Brasil.

\section{Jefferson Pedroso}

Graduando em Ciências Biológicas, Unochapecó - Chapecó (SC), Brasil.

\section{Maria Assunta Busato iD}

Doutora em Biologia, Universidade de Barcelona. Professora, Programa de Pós-Graduação em Ciências da Saúde, Unochapecó - Chapecó (SC), Brasil.

\section{Flávio Roberto}

\section{Mello Garcia (D)}

Doutor em Zoologia, Pontifícia Universidade Católica do Rio Grande do Sul. Professor, Programa de Pós-Graduação em Entomologia, Universidade Federal de PelotasPelotas (RS), Brasil.

Endereço para correspondência: Junir Antonio Lutinski-Rua Beija-Flor, 254 E, Efapi - CEP 89809-760 Chapecó (SC), Brasil E-mail:junir@unochapeco.edu.br

Recebido em: 22/10/2018

Aceito em: 03/12/2018

\section{RESUMO}

A formação de Áreas de Preservação Permanente (APPs) no entorno de lagos de usinas hidrelétricas (UHEs) ajuda a amenizar os impactos do empreendimento sobre a biodiversidade. Este estudo avaliou a riqueza, a abundância e a composição das assembleias de formigas que ocorrem na área de APP da UHE Foz do Chapecó. Foi realizada uma amostragem em cinco sítios, com históricos distintos de uso do solo, utilizando-se armadilhas pitfalls, guarda-chuva entomológico, rede de varredura, iscas de sardinha e de glicose e coleta manual. As assembleias foram avaliadas segundo a riqueza, a abundância e o índice de diversidade (H). Foram registradas 122 espécies pertencentes a 36 gêneros. A maior riqueza encontrada foi para Myrmicinae $(S=41)$ e Pheidole $(S=20)$, e a maior abundância, para Pheidole aberrans Mayr, 1868 ( $n=46)$. A riqueza pode ser $60,5 \%$ maior do que a observada. A riqueza, a abundância e a composição da fauna de formigas informa sobre o processo de reconstituição da APP.

Palavras-chave: bioindicadores; Mata Atlântica; mirmecofauna; riqueza; produção de energia.

\section{ABSTRACT}

The formation of Permanent Preservation Areas (APPs) around lakes of hydroelectric power plants (HPP) helps mitigate the impacts on biodiversity. This study evaluated the richness, abundance and composition of ant assemblies at the Foz do Chapecó's (HPP) permanent preservation area. Sampling was carried out at five sites, with distinct land use histories, using pitfalls, entomological umbrella, sweep net, sardine and glucose baits, and manual collection. Assemblies were evaluated according to richness, abundance and diversity index (H). 122 species belonging to 36 genera were recorded. The greatest richness found was for Myrmicinae $(S=41)$ and Pheidole $(S=20)$ and the highest abundance for Pheidole aberrans Mayr, 1868 ( $n=46)$. The richness may be $60.5 \%$ higher than the observed. The richness, abundance and composition of the ant fauna informs about the process of permanent preservation area's reconstitution.

Keywords: bioindicators; Atlantic Forest; mymecofauna; richness; energy production. 


\section{INTRODUÇÃO}

O Brasil concentra uma das maiores biodiversidades do mundo (RIBAS et al., 2012), porém atividades como agricultura, pecuária, instalações industriais, produção de energia e urbanização causam impacto sobre os biomas e, consequentemente, representam ameaças à sustentabilidade ambiental e à conservação de espécies sensíveis às alterações (GARDNER, 2010). A demanda pela produção de energia cresce diretamente associada à preocupação com os impactos ambientais provenientes desse processo (SAAD et al., 2017). Decorrentes da construção de barragens ocorrem a supressão de vegetação, o desalojamento de espécies, o revolvimento e a compactação do solo (KLIEMANN \& DELARIVA, 2015).

Com base no Código Florestal Brasileiro (BRASIL, 2012), rios e lagos devem ser protegidos nas suas margens por uma faixa de vegetação, variável em largura de acordo com do curso hídrico em questão. $O$ isolamento de Área de Preservação Permanente (APP) para regeneração em bordas de lagos formados a partir da instalação de usinas hidrelétricas (UHEs) não evita a perda de hábitats, o desalojamento de espécies e as alterações microclimáticas locais decorrentes da instalação do empreendimento. Contudo, o ambiente adjacente ao lago tende a evoluir para uma nova condição de equilíbrio com o passar do tempo. Nesse contexto, a APP pode contribuir positivamente para que condições próximas ao original se reestabeleçam no entorno (CAMPAGNOLO et al., 2017).

As formigas apresentam elevada riqueza e são ecologicamente importantes nos diferentes estratos dos ecossistemas terrestres (BACCARO et al., 2015). O estudo sobre assembleias de formigas é uma das técnicas utilizadas para avaliar mudanças no ambiente, enquadrando-se como uma ferramenta de avaliação das condições ambientais, além de proporcionar o monitoramento de áreas degradadas ou em condições de regeneração (NYAMUKONDIWA \& ADDISON, 2014). $O$ potencial bioindicador se deve às associações íntimas das formigas com a flora e com os demais grupos de invertebrados, bem como fornece estimativas do estágio de conservação ambiental (LUTINSKI et al., 2014; LUTINSKI et al., 2016) e da complexidade estrutural do habitat (ARMBRECHT; PERFECTO; VANDERMEER, 2004). O crescimento do número de estudos desenvolvidos com a finalidade de mostrar o potencial bioindicador da mirmecofauna se deve ainda à ampla distribuição geográfica, à rápida resposta às mudanças ambientais e à relevância biológica desses insetos nos diferentes níveis tróficos (CREPALDI et al., 2014; BHARTI; BHARTI; PFEIFER, 2016; LUTINSKI et al., 2017).

O conhecimento sobre a riqueza e abundância das assembleias de formigas em um determinado ambiente pode subsidiar planos de manejo (LUTINSKI et al., 2017). Informações sobre as interações das formigas com outros organismos possibilita predizer a biodiversidade associada e delinear estratégias para a conservação (CREPALDI et al., 2014; LUTINSKI et al., 2014). Na região onde uma UHE é instalada, a APP possibilita a colonização por espécies pioneiras no início do processo de recuperação ambiental (KWON; LEE; SUNG, 2014), culminando no restabelecimento da flora e da fauna (ULYSHEN, 2011). Dada a necessidade de monitoramento dos impactos ambientais causados pela instalação de UHE e, sobretudo, da contribuição da APP para o restabelecimento de uma nova condição de equilíbrio do ecossistema afetado, emerge a demanda pelo biomonitoramento de espécies indicadoras. Organismos que, pela riqueza, abundância, resiliência e produtividade, permitem estabelecer predições sobre o estágio de regeneração ao longo do tempo (RAPPORT; CONSTANZA; MCMICHAEL, 1998; RIBAS et al., 2012).

A Usina Hidrelétrica Foz do Chapecó é uma das maiores UHEs do Sul do Brasil, e a avaliação das comunidades de formigas na APP adjacente pode informar sobre a riqueza e distribuição de espécies em ambientes dos Estados de Santa Catarina e Rio Grande do Sul, servindo de base para estudos subsequentes. Nesse contexto, torna-se essencial conhecer a biodiversidade existente em ambientes com diferentes históricos de uso do solo e reconhecer padrões que ajudem na identificação de ambientes de interesse para a conservação. Considerando o crescente número de UHEs na Região Sul do Brasil nas últimas duas décadas e a contínua fragmentação do bioma Mata Atlântica, emerge a necessidade de compreender a dinâmica de regeneração em APPs adjacentes às hidrelétricas, além do potencial dessas áreas para a conservação da biodiversidade.

Nesse contexto, este estudo teve como objetivos:

- caracterizar as assembleias de formigas que ocorrem em sítios com diferentes históricos de uso do solo da APP; e

- avaliar a riqueza, a abundância e a composição das assembleias de formigas que ocupam diferentes nichos. 


\section{MATERIAIS E MÉTODOS}

\section{Área de estudo}

$O$ estudo foi realizado em cinco sítios da área de APP do reservatório da UHE Foz do Chapecó. A região está inserida no bioma Mata Atlântica (floresta ombrófila mista). O clima é do tipo superúmido mesotérmico subtropical úmido, sem estação seca definida e com distribuição regular da pluviosidade (RAMOS; SANTOS; FORTES, 2009).

A área do reservatório é de $79,2 \mathrm{~km}^{2}$ e está localizado na divisa entre os Estados de Santa Catarina e Rio Grande do Sul (FOZ DO CHAPECÓ, 2015). No período das amostras, o reservatório contava com sete anos desde a formação. Cada sítio amostrado conta com uma área de dois hectares, três estão localizados em Santa Catarina, municípios de Águas de Chapecó (27 14'51"S; $\left.53^{\circ} 03^{\prime} 55^{\prime \prime} \mathrm{W}\right)$, Caxambu do Sul $\left(27^{\circ} 15^{\prime} 35^{\prime \prime} \mathrm{S}\right.$; $\left.52^{\circ} 42^{\prime} 40^{\prime \prime} \mathrm{W}\right)$ e Guatambú ( $27^{\circ} 14^{\prime} 59^{\prime \prime} \mathrm{S}$; 52 $\left.41^{\prime} 06^{\prime \prime} \mathrm{W}\right)$; e dois no Rio Grande do Sul, município de Rio dos Índios ( $27^{\circ} 17^{\prime} 38^{\prime \prime}$ S; $52^{\circ} 44^{\prime} 58^{\prime \prime} \mathrm{W}$ e $27^{\circ} 20^{\prime} 41^{\prime \prime}$; ; 52 $43^{\prime} 51^{\prime \prime} \mathrm{W}$ ).

O sítio Ach está localizado no município de Águas de Chapecó. Trata-se do canteiro de obras e, entre os ambientes amostrados, representa aquele que sofreu o maior impacto. Durante a construção da UHE, toda a vegetação foi removida e o solo sofreu revolvimento e compactação pelas máquinas. Após a conclusão das obras, o sítio foi isolado por sete anos para regeneração natural. A vegetação, durante o período das amostras, era composta majoritariamente por gramíneas e por arbustos esparsos.

\section{Amostragem}

A amostragem foi conduzida nos meses de dezembro de 2017 e janeiro de 2018 em um único evento amostral. Foram utilizadas seis técnicas de amostragem: pitfalls, iscas de sardinha, iscas de glicose, guarda-chuva entomológico, rede entomológica e coleta manual. Um total de 10 pitfalls e 20 iscas (10 de cada) foi distribuído em transectos perpendiculares ao reservatório, equidistantes $20 \mathrm{~m}$ entre si, em cada sítio. As armadiIhas pitfall consistiram em copos plásticos de $250 \mathrm{~mL}$ (10 $\mathrm{cm}$ de diâmetro por $12 \mathrm{~cm}$ de altura), enterrados até a borda ao nível do solo. Em cada armadilha foram adicionados $150 \mathrm{~mL}$ de água com uma gota de detergente neutro. As iscas sardinha ( $1 \mathrm{~g})$ e glicose
O sítio Cax está localizado no município de Caxambu do Sul. Antes da formação do lago era uma área de cultivo agrícola. A vegetação é composta majoritariamente por gramíneas e arbustos esparsos com até seis metros de altura. A área é cortada por uma antiga estrada que dá acesso ao lago. Observa-se serrapilheira em formação.

O sítio Gua, localizado no município de Guatambú, conta com uma plantação de eucalipto (Eucalyptus grandis Hill ex Maiden) com idade de aproximadamente 12 anos. Quando da formação do lago, a vegetação já estava presente. É possível observar a presença de um sub-bosque, composto de vegetação nativa, com uma altura de até $6 \mathrm{~m}$. A serrapilheira está presente de forma descontínua.

O sítio Ri1 está situado no município de Rio dos Índios. Antes do isolamento como APP era coberto por pastagens. A vegetação é composta por árvores nativas que já existiam antes da formação do lago. Além dessa vegetação, o ambiente se encontra coberto por pequenos arbustos e a serrapilheira também está presente de forma irregular.

O sítio Ri2, também situado no município de Rio dos Índios, representa o ambiente mais conservado. Trata-se de um fragmento florestal, em estágio avançado de sucessão, composto por vegetação nativa e densa. Já existia há aproximadamente 20 anos antes da demarcação da APP. O solo é coberto por uma serrapilheira contínua.

( 1 $\mathrm{mL}$ ) foram dispostas sobre retângulos de papel de $20 \times 30 \mathrm{~cm}$, sobre o solo. Os pitfalls permaneceram no ambiente por 48 horas e as iscas, por 1 hora (LUTINSKI; LOPES; MORAIS, 2013).

O guarda-chuva entomológico consistiu em um retângulo de tecido com tamanho de $1 \mathrm{~m}^{2}$, suportado por um sarrafo de madeira em forma de $X$, preso nos quatro cantos. Em cada sítio, foi utilizado sob arbustos (10), escolhidos de forma aleatória na região aproximadamente central ao sítio. Os arbustos foram sacudidos por dez vezes cada um. Foi utilizada a Rede Entomológica de Varredura por um período de 30 minutos em 
cada sítio. As amostras, neste caso, foram obtidas sobre a vegetação rasteira até $1 \mathrm{~m}$ de altura em trilhas e clareiras.

Amostras manuais foram obtidas utilizando-se pinça e frasco com álcool (70\%). Ao todo, foi empregado um esforço amostral de uma hora de amostragem manual

\section{Identificação e análise de dados}

A identificação das amostras foi realizada seguindo as chaves propostas por Fernández (2003) e Baccaro et al. (2015). A classificação foi baseada em Bolton (2003). A riqueza foi definida como o número de espécies de formigas que ocorreram em cada uma das armadilhas, iscas, amostras com rede entomológica, guarda-chuva entomológico ou manualmente. A abundância foi definida com base na frequência relativa (registro de uma dada espécie em cada armadilha ou isca) (ROMERO \& JAFFE, 1989). A riqueza de cada assembleia foi comparada por meio de análise de rarefação baseada no número de ocorrências. Para tal, foi utilizado o programa EcoSim 7 (GOTELLI \& ENTSMINGER, 2001).

As assembleias de formigas foram comparadas quanto à riqueza, à abundância e aos índices de diversidade de Shannon-Weaver $\left(H^{\prime}\right)$ e de equitabilidade $\left(J^{\prime}\right)$. em cada sítio e esta foi realizada sobre a vegetação (até $1,8 \mathrm{~m}$ de altura).

Os espécimes amostrados foram transportados para o Laboratório de Entomologia da Universidade Comunitária da Região de Chapecó (Unochapecó) para triagem e identificação.

Também foram obtidas estimativas de riqueza (Chao 1) para cada sítio amostrado. Esses parâmetros foram obtidos com o auxílio do programa estatístico Past (HAMMER; HARPER; RYAN, 2001).

Foi utilizada uma ordenação Non-metric Multidimensional Scaling (NMDS) para testar se a abundância e a composição das assembleias de formigas diferem entre os sítios e quanto aos estratos em que foram amostradas. A matriz dos dados foi previamente transformada em Log $(x+1)$, foi utilizado Bray-Curtis como índice de associação e a análise foi realizada com o programa estatístico Primer 6.1.9. (CLARKE \& GORLEY, 2005). Nessa análise, foram juntadas as amostras das iscas de sardinha e de glicose por ambas contemplarem o mesmo estrato do ambiente. $\mathrm{O}$ mesmo procedimento foi adotado para as amostras da rede entomológica de varredura e do guarda-chuva.

\section{RESULTADOS}

Ao todo, foram registradas 541 ocorrências de formigas nas amostras, 122 espécies, pertencentes a oito subfamílias. O sítio Ri2 apresentou a maior riqueza $(S=59)$, seguido pelos sítios Ri1 $(S=45), C a x(S=38)$, Ach $(S=33)$ e Gua $(S=29)$ (Tabela 1). A diferença na riqueza entre cada sítio foi significativa (Figura 1). Relação semelhante à riqueza foi observada quanto à abundância: no sítio Ri2 foi obtido o maior número de ocorrências ( $n=122)$ e no sítio Gua, o menor $(n=83)$. $\mathrm{O} \mathrm{H}^{\prime}$ também apresentou maior valor $(3,81)$ no sítio Ri2, contudo o menor valor $(2,85)$ foi obtido para o sírio Ach. A equitabilidade J' foi similar nos sítios, variando entre 0,81 e 0,93. A maior estimativa (Chao $1=99,6$ ) foi encontrada para o sítio Ri2 e a menor, para o sítio Gua (Chao 1 = 42) (Tabela 1).

A subfamília Myrmicinae foi a mais rica nas amostras $(S=41)$, seguida por Formicinae $(S=28)$ e Ponerinae $(S=14)$. Os gêneros mais ricos foram Pheidole $(S=20)$ e Camponotus ( $S=18$ ). As espécies mais abundantes nas amostras foram Pheidole aberrans (Mayr, 1868) $(\mathrm{n}=46)$, Pachycondyla striata (F. Smith, 1858) ( $n=37)$, Nylanderia fulva (Mayr, 1862) $(\mathrm{n}=34)$, Pheidole lignicola (Mayr, 1887) ( $\mathrm{n}=26$ ), Pheidole punctatissima (Mayr, 1870) $(n=26)$ e Camponotus rufipes (Fabricius, 1775) $(n=19)$. Ao todo, 10 espécies ocorreram exclusivamente no sítio Gua: Camponotus novogranadensis (Mayr, 1870), Camponotus punctulatus (Mayr, 1868), Camponotus sp. 1, Labidus coecus (Latreille, 1802), Linepithema angulatum (Emery, 1894), Myrmicocrypta bruchi (Santschi, 1936), Neoponera vilosa (Fabricius, 1804), Pheidole sp. 2, Pheidole sp. 3 e Solenopsis sp. 1. Onze espécies ocorreram exclusivamente no sítio Cax: Camponotus melanoticus (Emery, 1894), Camponotus personatus (Emery, 1894), Cyphomyrmex plaumanni (Kempf, 1962), Ectatomma edentatum (Roger, 1863), Hypoponera trigona (Mayr, 1887), Labidus praedator (F. Smith, 1858), Linepithema iniquum (Mayr, 1870), 
Tabela 1 - Riqueza, frequência relativa das espécies, abundância, diversidade de Shannon-Weaver, equitabilidade e estimativas de riqueza (Chao 1) de formigas amostradas em cinco sítios da Área de Preservação Permanente formado pelo lago da Hidrelétrica Foz do Chapecó. Dezembro de 2017 e janeiro de 2018.

\begin{tabular}{|c|c|c|c|c|c|}
\hline Táxon & Gua & Cax & Ri1 & Ri2 & Ach \\
\hline \multicolumn{6}{|l|}{ Subfamília Dolichoderinae } \\
\hline \multicolumn{6}{|l|}{ Tribo Leptomyrmecini } \\
\hline Dorymyrmex brunneus (Forel, 1908) & & & & & 0,01 \\
\hline Linepithema angulatum (Emery, 1894) & 0,01 & & & & \\
\hline Linepithema gallardoi (Brèthes, 1914) & & & & 0,03 & \\
\hline Linepithema humile (Mayr, 1868) & 0,02 & 0,01 & & 0,01 & \\
\hline Linepithema inacatum (Bolton, 1969) & & & & 0,01 & \\
\hline Linepithema iniquum (Mayr, 1870) & & 0,02 & & & \\
\hline Linepithema micans (Forel, 1908) & & 0,01 & 0,01 & & \\
\hline Linepithema sp. 1 & & & 0,01 & 0,01 & \\
\hline Linepithema sp. 2 & & & 0,04 & & \\
\hline Linepithema sp. 3 & & & & 0,01 & \\
\hline
\end{tabular}

Subfamília Dorylinae

Labidus coecus (Latreille, 1802)

0,01

Labidus praedator (F. Smith, 1858)

0,02

Neivamyrmex punctaticeps (Emery, 1894)

0,01

Subfamília Ectatomminae

Tribo Ectatommini

Ectatomma edentatum (Roger, 1863)

0,01

Gnamptogenys striatula (Mayr, 1884)

0,01

0,06

0,02

Gnamptogenys striolata (Borgmeier, 1957)

0,01

\section{Subfamília Formicinae}

Tribo Camponotini

Camponotus alboannulatus (Mayr, 1887)

Camponotus cameranoi (Emery, 1894)

0,01

Camponotus crassus (Mayr, 1862)

0,04

Camponotus lespesii (Forel, 1886)

0,01

$0,01 \quad 0,01$ 
Tabela 1 - Continuação.

\section{Táxon}

Camponotus melanoticus (Emery, 1894)

Camponotus mus (Roger, 1863)

Camponotus novogranadensis (Mayr, 1870)

Camponotus punctulatus (Mayr, 1868)

Camponotus rufipes (Fabricius, 1775)

Camponotus sericeiventris (Guérin-Méneville, 1838)

Camponotus sexguttatus (Fabricius, 1793)

Camponotus sp. 1

Camponotus sp. 2

Camponotus sp. 3

Camponotus sp. 4

Camponotus sp. 5

Camponotus sp. 6

Camponotus sp. 7

Colobopsis personata (Emery, 1894)

Tribo Myrmelachistini

Brachymyrmex aphidicola (Forel, 1909)

Brachymyrmex coactus (Mayr, 1887)

Brachymyrmex cordemoyi (Forel, 1895)

Myrmelachista catharinae (Mayr, 1887)

Myrmelachista gagatina (Emery, 1894)

Tribo Lasiini

Nylanderia fulva (Mayr, 1862)

Nylanderia sp. 1

Nylanderia sp. 2

Paratrechina longicornis (Latreille, 1802)
Gua

Cax

Ri1

Ri2

Ach

0,01

$0,07 \quad 0,02$

0,01

0,02

0,01

0,01

0,01

0,08

0,03

0,05

0,02

0,01

0,01

0,01

\begin{tabular}{l|l}
\hline 0,01 & 0,01 \\
\hline 0,01 & \\
\hline 0,01 & \\
\hline
\end{tabular}

0,01

0,01

0,01

0,01

Subfamília Heteroponerinae

Tribo Heteroponerini

Heteroponera flava (Kempf, 1962)

$0,02 \quad 0,01$

Heteroponera inermis (Emery, 1894)

0,01

0,01

0,30

0,03

$0,01 \quad 0,02$

0,05

0,03

0,01

0,01

0,01

0,01

$0,01 \quad 0,01$


Tabela 1 - Continuação.

\begin{tabular}{|c|c|c|c|c|c|}
\hline Táxon & Gua & Cax & Ri1 & Ri2 & Ach \\
\hline \multicolumn{6}{|l|}{ Subfamília Myrmicinae } \\
\hline \multicolumn{6}{|l|}{ Tribo Attini } \\
\hline Acromyrmex ambiguus (Emery, 1888) & 0,01 & 0,02 & & & 0,01 \\
\hline Acromyrmex aspersus (F. Smith, 1858) & & & & 0,02 & \\
\hline Acromyrmex disciger (Mayr, 1887) & & & 0,02 & 0,01 & \\
\hline Acromyrmex subterraneus (Forel, 1893) & 0,02 & 0,02 & & & 0,01 \\
\hline Apterostigma mayri (Forel, 1893) & & 0,02 & & & 0,02 \\
\hline Apterostigma pilosum (Mayr, 1865) & & & 0,02 & 0,01 & \\
\hline Apterostigma wasmannii (Forel, 1892) & & & & 0,02 & \\
\hline Apterostigma sp. & & & 0,01 & 0,01 & \\
\hline Cephalotes angustus (Mayr, 1862) & & & & & 0,02 \\
\hline Cephalotes pusillus (Klug, 1824) & & 0,02 & 0,02 & 0,02 & 0,04 \\
\hline Cephalotes sp. 1 & & & 0,01 & & \\
\hline Cephalotes sp. 2 & & & & & 0,01 \\
\hline Cyphomyrmex plaumanni (Kempf, 1962) & & 0,01 & & & \\
\hline Cyphomyrmex strigatus (Mayr, 1887) & & & 0,03 & & \\
\hline Cyphomyrmex sp. & & & & 0,02 & \\
\hline Mycetosoritis sp. & & 0,01 & & & \\
\hline Myrmicocrypta bruchi (Santschi, 1936) & 0,01 & & & & \\
\hline Octostruma rugifera (Kempf, 1960) & & 0,01 & & & \\
\hline Pheidole aberrans (Mayr, 1868) & 0,11 & 0,17 & 0,05 & 0,09 & \\
\hline Pheidole dyctiota (Kempf, 1972) & & & 0,03 & 0,05 & \\
\hline Pheidole laevifrons (Mayr, 1887) & 0,04 & & 0,06 & 0,02 & \\
\hline Pheidole lignicola (Mayr, 1887) & 0,07 & 0,08 & 0,02 & 0,02 & 0,05 \\
\hline Pheidole megacephala (Fabricius, 1793) & 0,02 & & 0,02 & 0,02 & \\
\hline Pheidole pubiventris (Mayr, 1887) & & & 0,06 & 0,01 & \\
\hline Pheidole punctatissima (Mayr, 1870) & 0,07 & 0,09 & 0,02 & 0,02 & 0,05 \\
\hline Pheidole risii (Forel, 1892) & & & 0,07 & 0,03 & \\
\hline Pheidole tristis (F. Smith, 1858) & & & 0,01 & 0,05 & \\
\hline Pheidole sp. 1 & 0,04 & 0,05 & & & 0,06 \\
\hline
\end{tabular}


Tabela 1 - Continuação.

\begin{tabular}{|c|c|c|c|c|c|}
\hline Táxon & Gua & Cax & Ri1 & Ri2 & Ach \\
\hline Pheidole sp. 2 & 0,05 & & & & \\
\hline Pheidole sp. 3 & 0,02 & & & & \\
\hline Pheidole sp. 4 & & 0,01 & 0,01 & & \\
\hline Pheidole sp. 5 & & 0,02 & & 0,01 & \\
\hline Pheidole sp. 6 & & 0,01 & & 0,01 & \\
\hline Pheidole sp. 7 & & 0,01 & & & \\
\hline Pheidole sp. 8 & & & & 0,04 & \\
\hline Pheidole sp. 9 & & & & 0,02 & \\
\hline Pheidole sp. 10 & & & & 0,02 & \\
\hline Pheidole sp. 11 & & & & 0,01 & \\
\hline Procryptocerus adlerzi (Mayr, 1887) & & 0,01 & & 0,01 & \\
\hline Strumigenys cultrigera (Mayr, 1887) & & & & & 0,01 \\
\hline Wasmannia auropunctata (Roger, 1863) & & 0,01 & & 0,01 & \\
\hline Wasmannia sp. & & & 0,02 & & \\
\hline \multicolumn{6}{|l|}{ Tribo Crematogastrini } \\
\hline Crematogaster acuta (Fabricius, 1804) & & 0,01 & & & 0,02 \\
\hline Crematogaster corticicola (Mayr, 1887) & & & & 0,01 & 0,01 \\
\hline Crematogaster magnifica (Santschi, 1925) & & & & 0,01 & \\
\hline Crematogaster sp. & & & & & 0,02 \\
\hline \multicolumn{6}{|l|}{ Tribo Pogonomyrmecini } \\
\hline Patagonomyrmex angustus (Mayr, 1870) & & 0,02 & & 0,02 & \\
\hline Pogonomyrmex naegelii (Forel, 1878) & & & & & 0,07 \\
\hline \multicolumn{6}{|l|}{ Tribo Solenopsidini } \\
\hline Diplorhoptrum strictum (Emery, 1896) & & 0,05 & 0,03 & 0,03 & 0,02 \\
\hline Monomorium pharaonis (Linnaeus, 1758) & & 0,01 & & & 0,01 \\
\hline Monomorium floricola (Jerdon, 1851) & & 0,01 & 0,01 & & 0,04 \\
\hline Solenopsis saevissima (F. Smith, 1855) & & & & & 0,01 \\
\hline Solenopsis schmalzi (Forel, 1901) & 0,01 & & & & 0,04 \\
\hline Solenopsis sp. 1 & 0,01 & & & & \\
\hline Solenopsis sp. 2 & & & & 0,01 & \\
\hline
\end{tabular}


Tabela 1 - Continuação.

\begin{tabular}{|c|c|c|c|c|c|}
\hline Táxon & Gua & Cax & Ri1 & Ri2 & Ach \\
\hline \multicolumn{6}{|l|}{ Subfamília Ponerinae } \\
\hline \multicolumn{6}{|l|}{ Tribo Ponerini } \\
\hline Dinoponera australis (Emery, 1901) & & & & 0,02 & \\
\hline Hypoponera distinguenda (Emery, 1890) & 0,02 & & 0,01 & 0,01 & \\
\hline Hypoponera opaciceps (Mayr, 1887) & & & & 0,01 & \\
\hline Hypoponera trigona (Mayr, 1887) & & 0,01 & & & \\
\hline Hypoponera sp. 1 & & & & & 0,01 \\
\hline Hypoponera sp. 2 & & & & & 0,01 \\
\hline Neoponera crenata (Roger, 1858) & & & 0,01 & & \\
\hline Neoponera vilosa (Fabricius, 1804) & 0,01 & & & & \\
\hline Pachycondyla harpax (Fabricius, 1804) & & & & 0,02 & \\
\hline Pachycondyla striata (F. Smith, 1858) & 0,16 & 0,10 & 0,07 & 0,04 & \\
\hline Pachycondyla sp. 1 & & & & 0,01 & \\
\hline Pachycondyla sp. 2 & & & & 0,01 & \\
\hline Odontomachus affinis (Guérin-Méneville, 1844) & & & 0,01 & & \\
\hline Odontomachus chelifer (Latreille, 1802) & 0,01 & 0,06 & 0,07 & 0,01 & \\
\hline \multicolumn{6}{|l|}{ Subfamília Pseudomyrmecinae } \\
\hline \multicolumn{6}{|l|}{ Tribo Pseudomyrmecini } \\
\hline Pseudomyrmex flavidulus (F. Smith, 1858) & 0,01 & 0,02 & & 0,01 & 0,01 \\
\hline Pseudomyrmex gracilis (Fabricius, 1804) & 0,02 & & & 0,01 & \\
\hline Pseudomyrmex phyllophilus (F. Smith, 1858) & & & 0,02 & 0,04 & \\
\hline Pseudomyrmex schuppi (Forel, 1901) & & 0,01 & & & 0,01 \\
\hline Pseudomyrmex termitarius (F. Smith, 1855) & & & & & 0,01 \\
\hline Pseudomyrmex sp. 1 & & & & & 0,01 \\
\hline Pseudomyrmex sp. 2 & & & & & 0,01 \\
\hline Abundância & 83 & 124 & 107 & 122 & 105 \\
\hline Riqueza (Sobs) & 29 & 38 & 45 & 59 & 33 \\
\hline Shannon-Weaver $\left(\mathrm{H}^{\prime}\right)$ & 2,98 & 3,12 & 3,53 & 3,81 & 2,85 \\
\hline Equitabilidade $\left(J^{\prime}\right)$ & 0,88 & 0,86 & 0,93 & 0,93 & 0,81 \\
\hline Estimativa de riqueza (Chao 1) & 42,0 & 62,4 & 70,3 & 99,6 & 55,7 \\
\hline Relação Sobs e Chao 1 (\%) & 44,8 & 64,3 & 56,2 & 68,8 & 68,7 \\
\hline
\end{tabular}

Gua: Guatambú, SC; Cax: Caxambu, SC; Ri1 e 2: Rio dos Índios, RS; Ach: Águas de Chapecó, SC. 
Mycetosoritis sp., Nylanderia sp. 1, Octostruma rugifera (Kempf, 1960) e Pheidole sp. 7. Quinze espécies de forma exclusiva no sítio Ri1: Camponotus crassus (Mayr, 1862), Camponotus sericeiventris (Guérin-Méneville, 1838), Camponotus sericeiventris (Guérin-Méneville, 1838), Camponotus sexguttatus (Fabricius, 1793), Camponotus sp. 3, Camponotus sp. 4, Cephalotes sp. 1, Cyphomyrmex strigatus (Mayr, 1887), Gnamptogenys striolata (Borgmeier, 1957), Heteroponera inermis (Emery, 1894), Linepithema sp. 2, Myrmelachista catharinae (Mayr, 1887), Neivamyrmex punctaticeps (Emery, 1894), Neoponera crenata (Roger, 1858), Odontomachus affinis (Guérin-Méneville, 1844) e Wasmannia sp. Vinte e três espécies ocorreram exclusivamente no sítio Ri2: Acromyrmex aspersus (F. Smith, 1858), Apterostigma wasmannii (Forel, 1892), Brachymyrmex aphidicola (Forel, 1909), Camponotus cameranoi (Emery, 1894), Camponotus sp. 5, Camponotus sp. 6, Camponotus sp. 7, Crematogaster magnifica (Santschi, 1925), Cyphomyrmex sp., Dinoponera australis (Emery, 1901), Hypoponera opaciceps (Mayr, 1887), Linepithema gallardoi (Brèthes, 1914), Linepithema inacatum (Bolton, 1969), Linepithema sp. 3, Myrmelachista gagatina (Emery, 1894), Pachycondyla harpax (Fabricius, 1804), Pachycondyla sp. 1, Pachycondyla sp. 2, Pheidole sp. 10, Pheidole sp. 11, Pheidole sp. 8, Pheidole sp. 9 e Solenopsis sp. 2. No Ach, foram amostradas 15 espécies exclusivamente: Brachymyrmex coactus (Mayr, 1887), Brachymyrmex cordemoyi (Forel, 1895), Camponotus alboannulatus (Mayr, 1887), Cephalotes angustus (Mayr, 1862), Cephalotes sp. 2, Crematogaster sp., Dorymyrmex brunneus (Forel, 1908), Hypoponera sp. 1, Hypoponera sp. 2, Pogonomyrmex naegelii (Forel, 1878), Pseudomyrmex termitarius (F. Smith, 1855), Pseudomyrmex sp. 1, Pseudomyrmex sp. 2, Solenopsis saevissima (F. Smith, 1855) e Strumigenys cultrigera (Mayr, 1887) (Tabela 1).

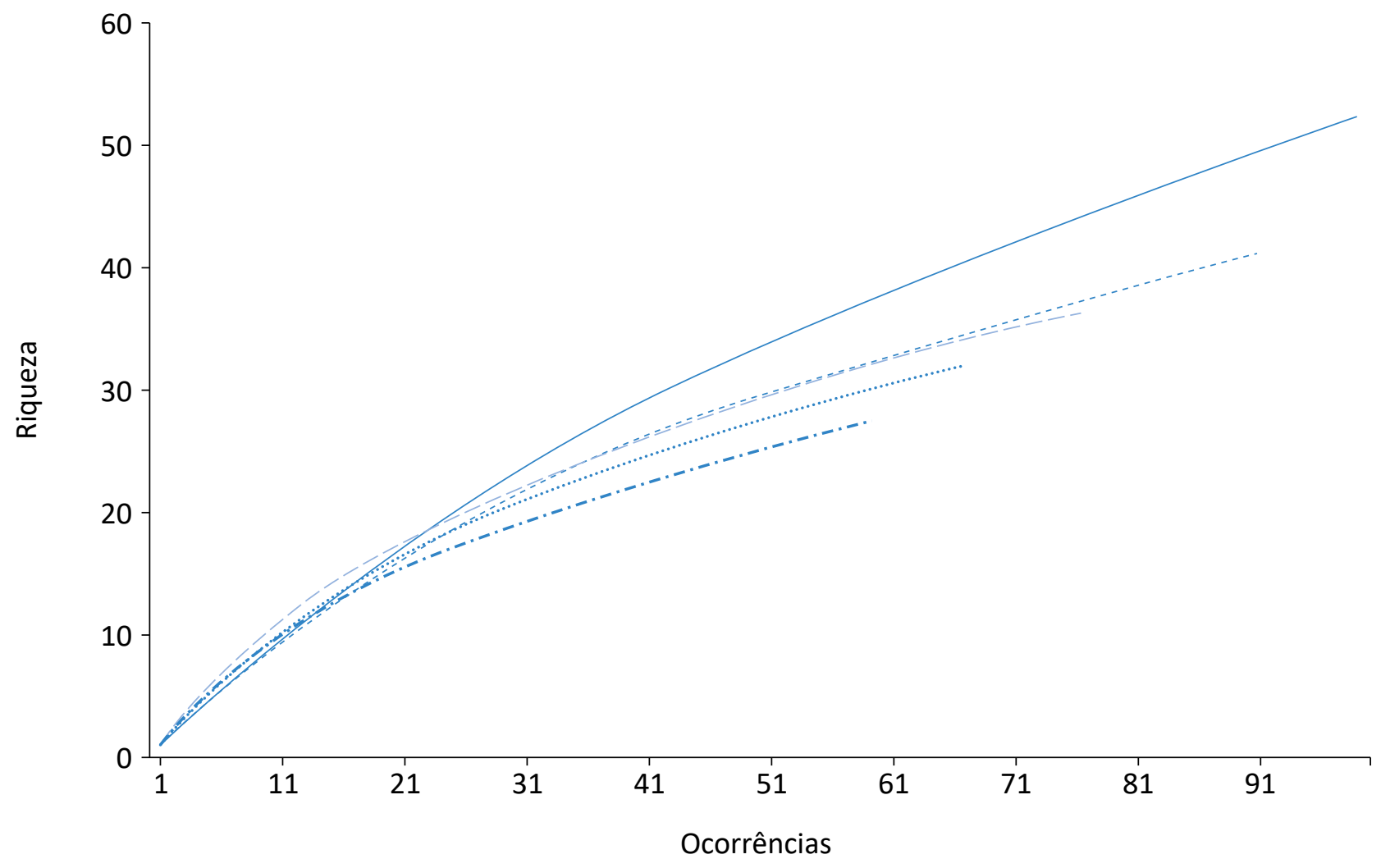

Ri2

Ri1

Cax

Ach

-.....-Gua

Gua: Guatambú, SC; Cax: Caxambu, SC; Ri1 e 2: Rio dos Índios, RS; Ach: Águas de Chapecó, SC.

Figura 1 - Comparativo, pelo método de rarefação baseado em ocorrências, das assembleias de formigas amostradas em cinco sítios da Área de Preservação Permanente formado pelo lago da Hidrelétrica Foz do Chapecó. Dezembro de 2017 e janeiro de 2018. 
A composição e a abundância diferiram nas amostras obtidas a partir de diferentes estratos dos sítios. Ao todo, 11 agrupamentos com similaridade de $35 \%$ entre si foram formados pela análise NMDS. As amostras obtidas nos pitfalls e nas iscas de sardinha e com glicose nos sítios Gua e Cax foram similares entre si. No sítio Ach, amostras com essas técnicas também apresentaram similaridade. Nos sítios Ri1 e Ri2, houve similaridade entre as amostras dos pitfalls e das iscas, contudo em dois grupos separados. As amostras obtidas com guarda-chuva entomológico e rede de varredura e as amostras manuais diferiram entre si e entre os ambientes, apresentando agrupamen- tos difusos entre os ambientes ou isolados das amostras (Figura 2).

O percentual de espécies registradas no solo e na serrapilheira (em pitfalls e iscas) foi maior $(82,2 \%)$ no sítio Ri1 e menor no sítio Ach (57,6\%). A riqueza amostrada associada à vegetação (guarda-chuva entomológico e rede de varredura e amostras manuais) foi percentualmente maior no sítio Ach $(21,2 \%)$ e menor no sítio Ri1 (13,3\%). Espécies amostradas concomitantemente no solo e na vegetação foram percentualmente maiores no sítio Ach $(21,2 \%)$ e menores no sítio Ri2 (3,4\%) (Figura 3).

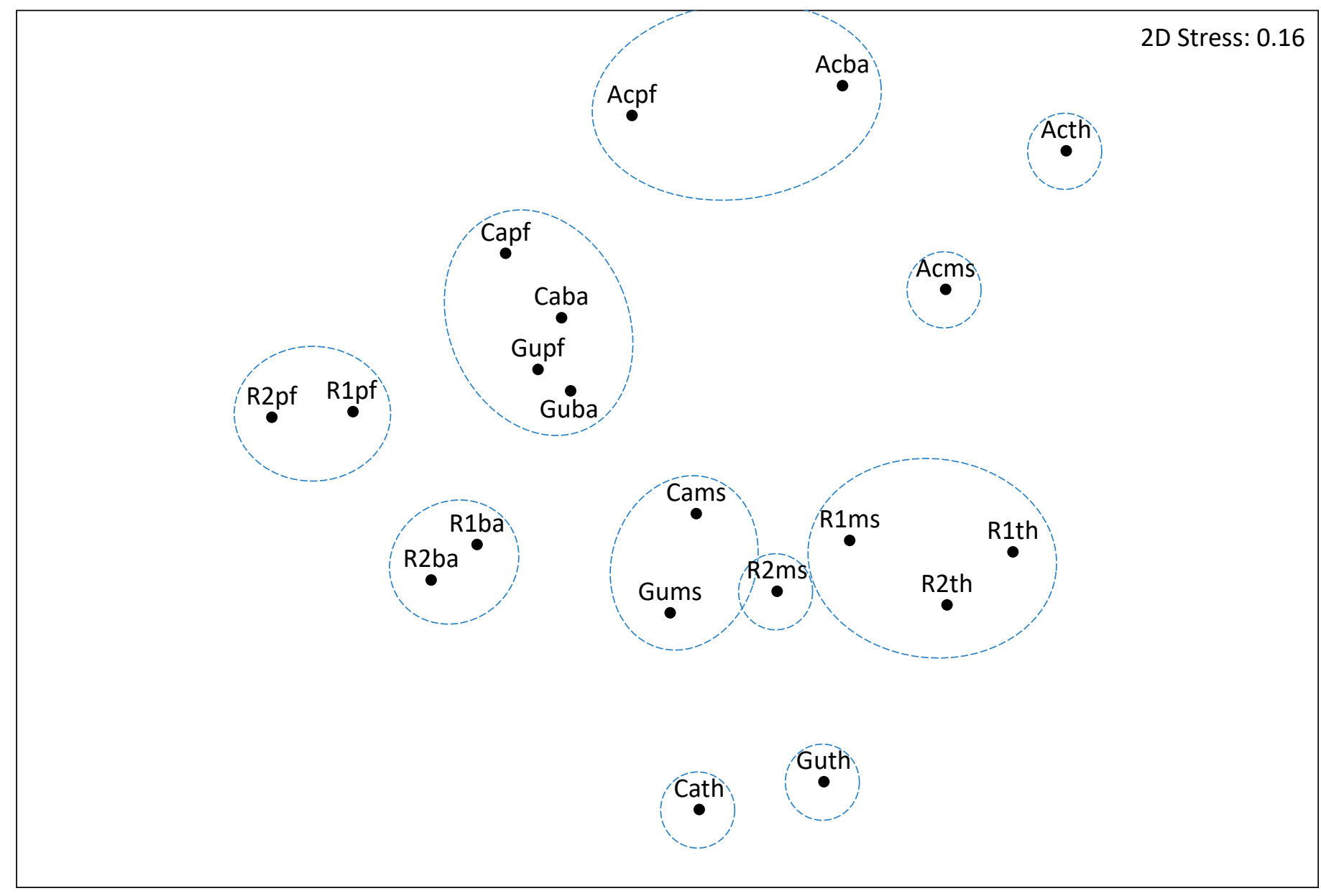

Gu: Guatambú, SC; Ca: Caxambu, SC; R1 e R2: Rio dos Índios, RS; Ac: Águas de Chapecó, SC; pf: pitfall, ba: iscas de sardinha e iscas de glicose; th: guarda-chuva entomológico e rede de varredura; ms: amostra manual. Associação pelo índice de Bray-Curtis. Os círculos representam 35\% de similaridade.

Figura 2 - Ordenação, pelo método Non-metric Multidimensional Scaling, das amostras de formigas, obtidas com diferentes técnicas, em cinco sítios da Área de Preservação Permanente formado pelo lago da Hidrelétrica Foz do Chapecó, dezembro de 2017 e janeiro de 2018. 


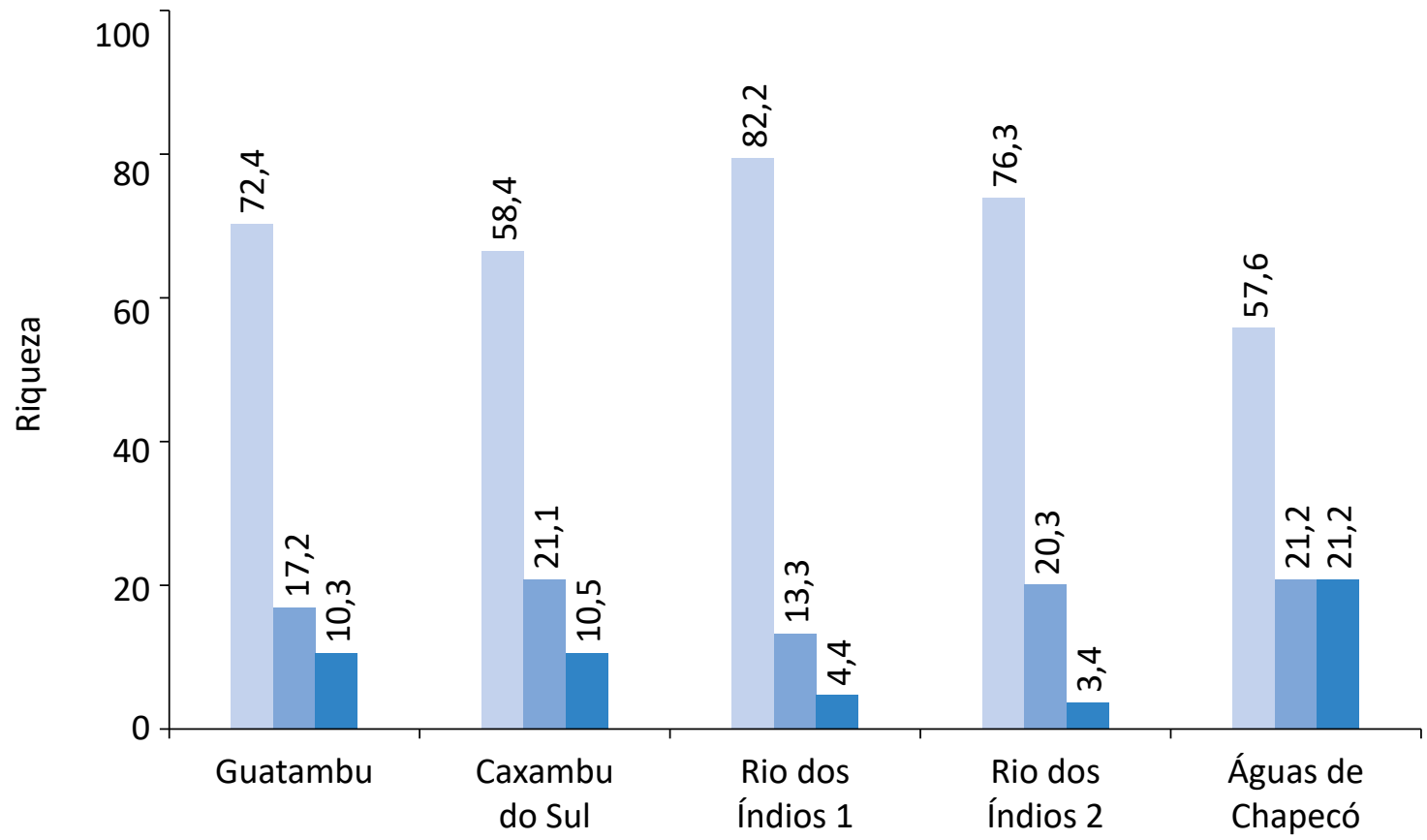

Sítios amostrados

Solo

Vegetação

Compartilhadas

Figura 3 - Distribuição da riqueza de formigas nas amostras segundo as técnicas de amostragem utilizadas em cinco sítios da Área de Preservação Permanente formado pelo lago da Hidrelétrica Foz do Chapecó, dezembro de 2017 e janeiro de 2018.

\section{DISCUSSÃO}

Os sítios em processo de regeneração mais avançado (Ri2) e com vegetação nativa (Cax, Ri1 e Ri2) apresentaram maior riqueza, abundância e diversidade $\left(\mathrm{H}^{\prime}\right)$ em relação ao sítio que sofreu o maior impacto (Ach) e ao sítio coberto por reflorestamento de eucalipto (Gua). A maior diferença $(68,8 \%)$ entre a riqueza amostrada e a estimada (Chao 1) foi observada no sítio Ri2, seguido pelo sítio mais impactado, ACH. A diversidade amostrada (subfamílias, gêneros e espécies) tem sido frequentemente registrada em inventários da mirmecofauna já realizados na Região Sul do Brasil (ULYSSÉA et al., 2011; CANTARELLI et al., 2015; LUTINSKI et al., 2017).

As subfamílias Myrmecinae e Formicinae são as mais ricas nos inventários da mirmecofauna realizados no bioma Mata Atlântica, tanto em número de gêneros quanto de espécies (ULYSSÉA et al., 2011; BACCARO et al., 2015). A diversidade desses táxons está relacionada aos hábitos alimentares diversificados que apresentam, aos diferentes nichos que ocupam e às especializa- ções que apresentam (HÖLLDOBLER \& WILSON, 1990). A subfamília Ponerinae também se destaca pela riqueza e abundância nos estudos realizados no Sul do Brasil. Caracteriza-se pelo hábito predador, o que indica diversidade de presas no ambiente, especialmente invertebrados (ULYSSÉA et al., 2011; BACCARO et al., 2015).

O gênero Pheidole apresentou a maior riqueza $(S=20)$. Formigas pertencentes aos gêneros Pheidole, Crematogaster, Solenopsis e Wasmannia são descritas na literatura como epígeas, onívoras e dominantes (SILVESTRE; BRANDÃO; SILVA, 2003). Esses gêneros são diversos, com distribuição nos diferentes biomas brasileiros (BACCARO et al., 2015). A riqueza desses gêneros indica estágio de regeneração ou conservação. Menor riqueza e maior abundância nas amostras indicam maior impacto, já que se observa tolerância em algumas espécies. $O$ aumento da riqueza indica estágio mais avançado de recuperação, visto que muitas espécies também ocorrem associadas à vegetação e à serapilheira (LUTINSKI et al., 2014; 2017; 2018). A riqueza 
de formigas desses gêneros indica avanço no processo de regeneração da APP, sete anos após a formação do lago. O caráter de onivoria e o hábito generalista também é atribuído às formigas Brachymyrmex e Nylanderia. O comportamento de recrutamento e o tamanho pequeno $(<2 \mathrm{~mm})$ dos espécimes (BACCARO et al., 2015) favorecem o acesso às fontes de alimento e podem explicar a ocorrência associada ao ambiente $\mathrm{ACH}$. A resiliência observada nas espécies desses gêneros as credencia como indicadoras de ambientes sob maior impacto, já que decresceram em abundância e riqueza nos sítios em estágio mais avançado de regeneração.

A riqueza de Dorymyrmex e de Linepithema é frequentemente descrita em associação com ambientes antropizados (ULYSSÉA et al., 2011). A dominância em ambientes ecologicamente perturbados é frequentemente relatada (SILVESTRE; BRANDÃO; SILVA, 2003; LUTINSKI et al., 2014). Os registros de espécies desses gêneros foram mais frequentes nos sítios Cax, Ri1 e Ri2, mostrando que as espécies inventariadas indicam mais fielmente ambientes em estágio intermediário de regeneração do que estágios mais iniciais, como é o caso de Gua e Ach.

Formigas do gênero Camponotus, segundo mais rico neste estudo $(S=18)$, ocorrem nos diferentes estratos do ambiente, desde o solo até a copa das árvores. $O$ mutualismo com insetos sugadores é frequentemente observado nessas formigas (SILVESTRE; BRANDÃO; SILVA, 2003). Algumas espécies são encontradas em ambientes antropizados (LUTINSKI et al., 2017), contudo o aumento na riqueza indica diversidade de vegetação e de nichos disponíveis (LUTINSKI \& GARCIA, 2005). A ausência de um padrão nas ocorrências dessas formigas nos sítios amostrados permite inferir que o potencial como descritores do estágio de regeneração está associado às espécies, e não ao gênero. Todos os sítios apresentaram espécies de Camponotus de forma exclusivas e, ao mesmo tempo, compartilharam C. mus.

Conhecidas como formigas cultivadoras de fungos, Acromyrmex, Apterostigma e Cyphomyrmex indicam a presença de massa vegetal, viva ou na forma de serapiIheira. Exercem papel na manutenção do solo em que as galerias dos seus ninhos contribuem para a aeração e a infiltração da água, enquanto seus excrementos o enriquecem (SILVESTRE; BRANDÃO; SILVA, 2003). Apterostigma e Cyphomyrmex ocorreram nos ambientes Ri1 e Ri2, mais conservados, e isso pode ser explicado pela presença da serapilheira nesses sítios, nos quais cultivam seus fungos. Espécies de Acromyrmex foram registradas também nos demais sítios, indicando tolerância das espécies amostradas e avanço no processo de regeneração da APP.

O hábito predador é compartilhado por formigas pertencentes aos gêneros Dinoponera, Gnamptogenys, Heteroponera, Hypoponera, Labidus, Neivamyrmex, Neoponera, Pachycondyla, Odontomachus e Strumigenys (BACCARO et al., 2015). A riqueza e a distribuição das ocorrências dessas formigas nas amostras indicam que, apesar das diferenças no estágio de regeneração em que os sítios se encontram, a APP, após sete anos de isolamento, oferece nichos comparáveis aos ambientes conservadas existentes na região (LUTINSKI et al., 2018). O crescimento da vegetação e a formação de serrapilheira em que essas formigas constroem seus ninhos e forrageiam (SILVESTRE; BRANDÃO; SILVA, 2003) podem explicar a riqueza amostrada.

Formigas Cephalotes, Procryptocerus e Pseudomyrmex são encontradas associadas à vegetação (BACCARO et al., 2015). A riqueza desses táxons no estudo caracteriza a regeneração da vegetação e a oferta de uma variedade de nichos para a nidificação e para o forrageio. Já as formigas Pogonomyrmex $(S=2)$ são coletoras de sementes (SILVESTRE; BRANDÃO; SILVA, 2003) e frequentes em ambientes abertos (LUTINSKI et al., 2014). A presença de formigas desses gêneros indica o avanço na recomposição da vegetação na APP.

A riqueza de formigas na APP pode ser, em média, $60,5 \%$ (Chao 1) maior do que a amostrada. A dinâmica na oferta de nichos durante o processo de regeneração da APP pode explicar a ocorrência de espécies em unicatas ou duplicatas, influenciando no resultado da estimativa (CHAO et al., 2009). É evidente também que, apesar da combinação de técnicas utilizadas e do esforço amostral empregado no inventário, uma amostragem apenas não é suficiente para inventariar toda a mirmecofauna da APP.

$\mathrm{O} \mathrm{H}^{\prime}$ variou entre 2,85 (Ach) e 3,81 (Ri2). Considerando-se o estágio de regeneração em que cada sítio se encontra, pode-se afirmar que $\mathrm{o}^{\prime} \mathrm{H}^{\prime}$ caracteriza um gradiente do sítio mais rico e em estágio mais avançado de regeneração, decrescendo para a área com histórico de pastagem, lavoura e reflorestamento, bem como para o canteiro de obras. Ainda, a diversidade $\mathrm{H}^{\prime}$ amostrada na APP é semelhante aos valores encontrados em ambientes conservados na região (LUTINSKI et al., 2008). A J' foi superior a 0,5 em todos os sítios, indicando, segundo Peralta e Martínez (2013), uniformidade na distribuição das ocorrências nas amostras e menor dominância. 
As amostras de formigas diferiram entre os sítios quanto à riqueza de formigas. A análise de rarefação apontou a maior riqueza no sítio Ri2, fragmento florestal, decrescendo para Ri1, Cax, Ach e Gua, um gradiente semelhante ao apontado pelo $\mathrm{H}^{\prime}$. O resultado corrobora o estudo realizado por Lutinski et al. (2018) sobre a importância de fragmentos florestais; contudo, indica que sete anos após a formação do lago, o histórico de uso do solo anterior ao isolamento para regeneração da APP ainda pode ser percebido a partir da riqueza de formigas.

As comunidades de formigas são afetadas pelos processos de regeneração da vegetação (RIBAS et al., 2012), onde a intensidade da degradação ou alterações no ambiente podem produzir respostas diversas, beneficiando algumas espécies e prejudicando outras (HÖLLDOBLER \& WILSON, 1990). A diversidade de formigas tende a aumentar de acordo com a complexidade dos ambien- tes, devido a maior disponibilidade de nichos presentes (HOLDEFER; LUTINSKI; GARCIA, 2017). As amostras deste estudo foram coletadas com o uso de um conjunto de métodos visando contemplar diferentes estratos dos ambientes. Nota-se que a composição e a abundância das espécies difere (NMDS) do solo e da serrapilheira (pitfall e iscas) para a vegetação (rede entomológica, guarda-chuva entomológico e amostras manuais). Nota-se também que um padrão de oferta e de ocupação dos nichos em cada sítio (Figura 3). Novamente, o sítio Ri3, mais conservado, apresentou maiores percentuais de espécies, ocorrendo apenas no solo ou na vegetação, e menor percentual da riqueza, ocorrendo de forma compartilhada. No outro extremo, o sítio Ach, submetido a maior impacto durante a implantação da UHE, apresentou menores percentuais de espécies, ocorrendo de forma exclusiva no solo ou vegetação e com um maior percentual de espécies sendo amostrado nos dois estratos.

\section{CONCLUSÃO}

Assembleias de formigas apresentam sensibilidade ao processo de regeneração da APP isolada para regeneração. Sítios em processo de regeneração mais avançado, com histórico prévio de cobertura por vegetação nativa, apresentam maior riqueza, abundância e diversidade em relação àqueles que sofrem maior impacto durante a instalação do empreendimento. Emerge a importância de planos de monitoramento pós-implantação de áreas diretamente afetadas por UHEs que contemplem ambientes com diferentes históricos de uso do solo. Ressalta-se também a importância da utilização de um protocolo de amostragem com diferentes técni- cas que contemplem os diferentes estratos do solo e da vegetação.

A diversidade amostrada (subfamílias, gêneros e espécies) tem sido frequentemente registrada em inventários da mirmecofauna já realizados na Região Sul do Brasil. Esse aspecto aponta para o potencial do uso das formigas como bioindicadores da diversidade e como descritores para o monitoramento pós-implantação de empreendimentos como UHE. Os resultados apontam que assembleias de formigas respondem ao processo de reconstituição da APP. A riqueza, a abundância e a composição das assembleias são preditores do estágio de regeneração dos estratos formados.

\section{AGRADECIMENTOS}

Ao Consórcio Foz do Chapecó, pela permissão para a amostragem na APP. À Universidade Comunitária da Região de Chapecó (UNOCHAPECÓ), pela bolsa de pesquisa iniciação científica (edital n³5/REITORIA/2018).
A realização do estudo foi autorizada pelo Instituto Chico Mendes de Conservação da Biodiversidade (ICMBio) - "autorização para atividades com finalidade científica" n 50739-2, de 16 de setembro de 2016.

\section{REFERÊNCIAS}

ARMBRECHT, I.; PERFECTO, I.; VANDERMEER, J. Enigmatic biodiversity correlations: ant diversity responds to diverse resources. Science, v. 304, n. 5668, p. 284-286, 2004. http://doi.org/10.1126/science.1094981

BACCARO, F. B.; FEITOSA, R. M.; FERNANDEZ, F.; FERNÁNDES, O. M.; IZZO, T.; SOUZA, J. L. P.; SOLAR, R. Guia para gêneros de formigas no Brasil. Manaus: INPA, 2015. 388 p. 
BHARTI, H.; BHARTI, M.; PFEIFER, M. Ants as bioindicators of ecosystem health in Shivalik Mountains of Himalayas: assessment of species diversity and invasive species. Asian Myrmecology, v. 8, p. 65-79, 2016. http://doi.org/10.20362/am.008023

BOLTON, B. Synopsis and classification of Formicidae. Gainesville: The American Entomological Institute, 2003. 370 p.

BRASIL. Código Florestal. Lei no 12.651, de 25 de maio de 2012. Diário Oficial da União, Brasília, 2012. Disponível em: <http://www.planalto.gov.br/ccivil_03/_ato2011-2014/2012/lei/l12651.htm>. Acesso em: 20 set. 2017.

CAMPAGNOLO, K.; SILVEIRA, G. L.; MIOLA, A. C.; SILVA, R. L. L. Área de preservação permanente de um rio e análise da legislação de proteção da vegetação nativa. Ciência Florestal, v. 27, n. 3, p. 831-842, 2017. http://dx.doi. org/10.5902/1980509828633

CANTARELLI, E. B.; FLECK, M. D.; GRANZOTTO, F.; CORASSA, J.; D’AVILA, M. Diversidade de formigas (Hymenoptera: Formicidae) da serrapilheira em diferentes sistemas de uso do solo. Ciência Florestal, v. 25, n. 3, p. 607-616, 2015. http://dx.doi.org/10.5902/1980509819612

CHAO, A.; COLWELL, R. K.; LIN, C. W.; GOTELLI, N. J. Sufficient sampling for asymptotic minimum species richness estimators. Ecological Society of America, v. 90, n. 4, p. 1125-1133, 2009. https://doi.org/10.1890/07-2147.1

CLARKE, K. R.; GORLEY, R. N. Primer: Getting started with v6. Plymouth routines in multivariate ecological research. Plymouth: Primer-E Ltd., 2005.

CREPALDI, R. A.; PORTILHO, I. I. R.; SILVESTRE, R.; MERCANTE, F. M. Formigas como bioindicadores da qualidade do solo em sistema integrado lavoura-pecuária. Ciência Rural, v. 44, n. 5, p. 781-787, 2014. http://dx.doi.org/10.1590/S010384782014000500004

FERNÁNDEZ, F. Introducción a las hormigas de la región neotropical. Bogotá: Instituto de Investigación de Recursos Biológicos Alexander von Humboldt, 2003. 433 p.

FOZ DO CHAPECÓ. Informações online sobre o consórcio da Hidrelétrica Foz do Chapecó. Consórcio Foz do Chapecó, Florianópolis, 2015. Disponível em: <http:www.fozdochapeco.com.br>. Acesso em: 18 set. 2017.

GARDNER, T. A. Monitoring forest biodiversity: improving conservation through ecologically-responsible management. Londres: Earth Scan, 2010. 360 p.

GOTELLI, N. J.; ENTSMINGER, G. L. EcoSim: Null models software for ecology. Versão 7.0. Acquired Intelligence Inc. \& Kesey-Bear, 2001. Disponivel em: <http://garyentsminger.com/ecosim/>. Acesso em: 18 set. 2017.

HAMMER, O.; HARPER, D. A. T.; RYAN, P. D. Past: Palaeonthological statistics software package for education and data analysis. Versão. 1.37. Palaeontologia Electronica, v. 4, n. 1, p. 1-9, 2001. Disponível em: <http://palaeo-electronica. org/2001_1/past/past.pdf>. Acesso em: 19 set. 2017.

HOLDEFER, D. R.; LUTINSKI, J. A.; GARCIA, F. R. M. Does organic management of agroecosystems contribute to the maintenance of the richness of ants? Semina: Ciências Agrárias, v. 38, n. 6, p. 3455-3468, 2017. http://doi. org/10.5433/1679-0359.2017v38n6p3455

HÖLLDOBLER, B.; WILSON, E. O. The Ants. Cambridge: Harvard University Press, 1990. 746 p.

KLIEMANN, B. C. K.; DELARIVA, R. L. Pequenas centrais hidrelétricas: cenários e perspectivas no estado do Paraná. Ciência e Natura, v. 37, n. 3, p. 274-283, 2015.

KWON, T. S.; LEE, C. M.; SUNG, J. H. Diversity decrease of ant (Formicidae, Hymenoptera) after a forest disturbance: different responses among functional guilds. Zoological Studies, v. 3, n. 53, p. 37-47, 2014. https://doi.org/10.1186/s40555-014-0037-z

LUTINSKI, J. A.; BAUCK, E. L.; FILTRO, M.; BUSATO, M. A.; KNAKIEWICZ, A. C.; GARCIA, F. R. M. Ant assemblage (Hymenoptera: Formicidae) in three wind farms in the State of Paraná, Brazil. Brazilian Journal of Biology, v. 1, n. 77, p. 176-184, 2016. http://dx.doi.org/10.1590/1519-6984.14115 
LUTINSKI, J. A.; GARCIA, F. R. M. Análise faunística de Formicidae (Hymenoptera: Apocrita) em ecossistema degradado no município de Chapecó, Santa Catarina. Biotemas, v. 18, n. 2, p. 73-86, 2005. https://doi.org/10.5007/\%25x

LUTINSKI, J. A.; GARCIA, F. R. M.; LUTINSKI, C. J.; IOP, S. Diversidade de formigas na Floresta Nacional de Chapecó, Santa Catarina, Brasil. Ciência Rural, v. 38, n. 7, p. 1810-1816, 2008. http://dx.doi.org/10.1590/S0103-84782008000700002

LUTINSKI, J. A.; ILHA, C.; LUTINSKI, C. J.; BAUCKE, L.; FILTRO, M.; BUSATO, M. A.; GARCIA, F. R. M. Ant fauna associated with areas under the direct impact of small hydropower plants in the state of Paraná, Brazil. Revista Brasileira de Ciências Ambientais, n. 46, p. 2-13, 2017. http://dx.doi.org/10.5327/Z2176-947820170196

LUTINSKI, J. A.; LOPES, B. C.; MORAIS, A. B. B. Urban ant diversity (Hymenoptera: Formicidae) in ten cities of southern Brazil. Biota Neotropica, v. 13, n. 3, p. 332-342, 2013. http://dx.doi.org/10.1590/S1676-06032013000300033

LUTINSKI, J. A.; LUTINSKI, C. J.; BELING, J. F.; BUSAT, M. A.; CORRALO, V. Ant assemblages (Hymenoptera: Formicidae) associated to environments of a rural property in the extreme western region of the state of Santa Catarina. Revista Brasileira de Ciências Ambientais, n. 47, p. 12-23, 2018. http://dx.doi.org/10.5327/Z2176-947820180180

LUTINSKI, J. A.; LUTINSKI, C. J.; LOPES, B. C.; MORAIS, A. B. B. Estrutura da comunidade de formigas (Hymenoptera: Formicidae) em quatro ambientes com diferentes níveis de perturbação antrópica. Ecología Austral, v. 24, n. 2, p. 229-237, 2014.

NYAMUKONDIWA, C.; ADDISON, P. Food Preference and Foraging Activity of Ants: Recommendations for Field Applications of Low-Toxicity Baits. Journal of Insect Science, v. 14, p. 48, 2014. https://dx.doi.org/10.1093\%2Fjis\%2F14.1.48

PERALTA, L.; MARTínEZ, P. A. Ensambles de ácaros oribátidos en hormigueros de Acromyrmex spp. (Hymenoptera, Formicidae). Ecología Austral, v. 23, n. 3, p. 209-217, 2013.

RAMOS, A. M.; SANTOS, L. A. R. dos; FORTES, L. T. G. (Orgs.). Normais Climatológicas do Brasil 1961-1990. Brasília: INMET, 2009. 465 p.

RAPPORT, D. J.; COSTANZA, R.; MCMICHAEL, A. J. Assessing ecosystem health. Trends in Ecology and Evolution, School of Biological Sciences, v. 13, n. 10, p. 397-402, 1998. https://doi.org/10.1016/S0169-5347(98)01449-9

RIBAS, C. R.; SCHMIDT, F. A.; SOLAR, R. R. C.; CAMPOS, R. B. F.; VALENTIM, C. L.; SCHOEREDER, J. H. Ants as indicators of the success of rehabilitation efforts in deposits of gold mining tailings. Restoration Ecology, v. 20, n. 6, p. 712-720, 2012. https://doi.org/10.1111/j.1526-100X.2011.00831.x

ROMERO, H.; JAFFE, K. A comparison of methods for sampling ants (Hymenoptera: Formicidae) in Savanna. Biotropica, v. 21 , n. 4 , p. 348-352, 1989. https://doi.org/10.2307/2388285

SAAD, L. P.; SOUZA-CAMPANA, D. R.; BUENO, O. C.; MORINI, M. S. Vinasse and Its Influence on Ant (Hymenoptera: Formicidae) Communities in Sugarcane Crops. Journal Insect Science, v. 17, n. 1, p. 11, 2017. https://dx.doi. org/10.1093\%2Fjisesa\%2Fiew103

SILVESTRE, R.; BRANDÃO, C. R. F.; SILVA, R. R. Grupos funcionales de hormigas: el caso de los gremios del Cerrado. In: FERNÁNDEZ, F. (Org.). Introducción a las hormigas de la región Neotropical. Bogotá: Instituto de Investigación de Recursos Biológicos Alexander von Humboldt, 2003. p. 113-148.

ULYSHEN, M. D. Arthropod vertical stratification in temperate deciduous forests: Implications for conservation-oriented management.ForestEcologyand Management, v.261, n.9, p.1479-1489,2011.https://doi.org/10.1016/j.foreco.2011.01.033

ULYSSÉA, M. A.; CERETO, C. E.; ROSUMEK, F. B.; SILVA, R. R.; LOPES, B. C. Updated list of ant species (Hymenoptera, Formicidae) recorded in Santa Catarina State, southern Brazil, with a discussion of research advances and priorities. Revista Brasileira de Entomologia, v. 55, n. 4, p. 603-611, 2011. http://dx.doi.org/10.1590/S0085-56262011000400018 